\title{
Capítulo
}

2

\section{Voz sobre IP (VoIP) - configurando servidor elastix para ensino-aprendizagem}

\author{
Ana Maura Pereira Cabral
}

\begin{abstract}
The Internet today is considered one of the largest engineering systems ever created, being considered "a network of networks". You can also describe it as an infrastructure that provides service applications. Thus, areas such as telecommunications have used technologies that proposes to use the Internet. And Voice over IP (VoIP) stands out for the importance of offering services such as telephony and voice transport using this network. However, its implementation requires solid knowledge about the theme and the elastix server is an interesting proposal in the teaching-learning process, as it is a free distribution of unified Communications Server integrating services such as VoIP and $P B X$. This chapter introduces the theory about VoIP, key features, and implementation of the elastix server.
\end{abstract}

\section{Resumo}

A Internet hoje é considerada uma dos maiores sistemas de engenharia já criado, sendo considerada "uma rede de redes". Também é possível descrevê-la como uma infraestrutura que provê aplicações de serviços. Assim, áreas como telecomunicações tem usado de tecnologias que propõe utilizar a Internet. E o Voz sobre IP (VoIP) se destaca pela importância em oferecer serviços como telefonia e transporte de voz. utilizando essa rede. Porém, a sua implementação exige conhecimentos sólidos acerca do tema e o servidor elastix é uma proposta interessante no processo de ensinoaprendizagem, por ser uma distribuição livre de servidor de comunicações unificadas integrando serviços como VoIP e PBX. Este capítulo apresenta a teoria sobre o VoIP, os principais recursos e a implementação do servidor elastix. 


\section{Introdução}

A telefonia sofreu mudanças a partir dos anos de 1990, seguindo tendências tecnológicas e principalmente, a evolução da Internet. Assim surgia os primeiros sistemas que integraram o Voz sobre IP (VoIP) ou Voicer over Internet Protocol (VoIP) do inglês, eram telefones convencionais que disseminavam esse tipo de tecnologia.

De acordo com Carvalho (2018) essa tecnologia "converte sinais de voz em pacotes os quais trafegam através da rede mundial de acesso livre IP, a internet, da mesma forma que os pacotes de dados, permitindo a comunicação por voz com qualidade", algumas vantagens são prover serviços como voz e dados em uma única infraestrutura e a redução de gastos. Visando promover qualidade e melhor comodidade aos usuários da rede.

Essa tecnologia alcançou público após a empresa VocalTec lançar o primeiro software comercial de VoIP, um telefone que permitia um usuário chamar outro usuário através da Internet no ano de 1995, em Israel, ficando conhecido como Internet Phone. No ano seguinte aplicações de correio de voz na Internet ganharam vida, porém, ainda nos anos seguintes, o público que utilizava dessa tecnologia era muito baixo. Algumas das problemáticas eram qualidade de som fraca, períodos de silêncio e perda de conexão.

Em 2005, o Skype apresentou um salto quando introduziram a chamada por vídeo em seu software. Tornou-se referência para outras empresas de VoIP no mercado. Com essa nova visão, medidas para correção das problemáticas existentes foram necessárias, surgindo os primeiros protocolos de sinalização para estabelecimento de chamadas de voz através de redes de pacotes voltados para esse tipo de sistema.

Essa tecnologia incorpora o conceito de serviço de qualidade e "uma rede está preparada para oferecer o serviço de VoIP quando ela possui o tratamento adequado para tal, desde permitir este tipo de tráfego através de seus firewalls até utilizar práticas de QoS para garantir a qualidade das ligações" (BORDIM, 2010).

Keller (2011, p. 20) afirma "com a ampliação das velocidades de acesso à Internet, o VoIP passou a fazer parte do dia a dia das grandes corporações, com o objetivo inicial e principal de reduzir o valor das contas telefônicas", neste contexto torna-se importante explorar o tema para o público acadêmico, apresentando as melhorias já alcançadas ao decorrer dos anos e também explorar esse universo rico e promissor no mercado.

O objetivo deste minicurso é apresentar os tópicos relevantes sobre o assunto Voz sobre IP, realizar a implementação do servidor elastix e configução dos ramais para ligações telefônicas, e por fim, utilizar de um softphone para emular telefone VoIP e assim verificar a veracidade dos ramais criado no servidor.

O restante do capítulo esta organizado da seguinte forma. A seção 2 apresenta os principais fundamentos sobre o VoIP. A seção 2.1 é destacado pela importância em dferenciar a telefonia convencional da telefonia VoIP. A seção 2.2 é apresentado os tipos de telefones VoIP que podem ser utilizados. A seção 2.3 como é a comunicação. A seção 2.4 o protocolo SIP utilizado para as principais aplicações desse tipo. A seção 3 é organizada para apresentar a metodologia utilizada neste minicurso. A seção 4 é apresentado os resultados obtidos com o desenvolvimento desse aparato. E por fim, a seção 5 apresenta as considerações sobre a realização deste minicurso. 


\section{Voz sobre IP(VoIP)}

\subsection{Telefonia Convencional $x$ Telefonia VoIP}

A telefonia convencional é permeada pelo conceito de uma rede de linhas analógicas e digitais, projetadas para estabelecer a comunicação de voz entre dois pontos. Essa rede também é utilizada para transmitir outros tipos de sinais analógicos, permitindo a comunicação entre pontos fixos e determinados. Enquanto, a telefonia VoIP faz referências às técnicas de empacotamento e transmissão de amostras de voz sobre redes IP e a mecanismos de sinalização para estabelecer as chamadas. As principais diferenças conforme Teleco (2019) é mostrado na tabela 1.

\section{Tabela 1 - Telefonia Convencional x VoIP}

\begin{tabular}{|l|l|l|}
\hline \multicolumn{1}{|c|}{ Característica } & \multicolumn{1}{c|}{ Telefonia Convencional } & Telefonia VoIP \\
\hline Conexão na casa do usuário & Cabo de cobre (par trançado) & Banda larga de Internet \\
\hline Falta de Energia Elétrica & Continua funcional & Para de funcionar \\
\hline Mobilidade & Limitada a casa do usuário & Acesso em qualquer lugar do mundo, desde que conectado a Internet \\
\hline Número Telefônico & Associado ao domicílio do usuário & Associado à área local do número contratado \\
\hline Chamadas locais & Área local do domicílio do usuário & Área local do número contratado \\
\hline
\end{tabular}

\subsection{Os tipos de telefones VoIP}

Para os serviços VoIP tornarem possíveis é necessário a utilização de telefones apropriados para as redes IP. De acordo com Teleco (2019), pode-se utilizar os seguintes tipos de telefones:

- Computador: o próprio computador pode ser usado como telefone IP, desde que tenha uma placa de som, um microfone, alto falantes ou fones de ouvidos, e um programa do tipo softphone, que possui todos os recursos para funcionar como um telefone IP.

- Adaptador para Telefone Analógico (ATA): é um dispositivo que funciona como um conversor de telefone IP para um telefone analógico convencional. O ATA é conectado a um acesso de banda larga (rede IP) e a um telefone analógico convencional, que pode ser usado normalmente para fazer e receber ligações do serviço VoIP contratado.

- Telefone IP: é um telefone que possui todos os recursos necessários para um serviço VoIP. Para ser usado é necessário apenas conectá-lo a um acesso de banda larga (rede IP) para fazer e receber ligações do serviço VoIP.

\subsection{Comunicação para telefonia VoIP}

A comunicação entre os dispositivos ocorre através de dois processos simultâneos, e conforme Teleco (2019) são: 
- Sinalização e Controle de Chamadas: estabelecimento da chamada, acompanhamento da chamada e finalização da chamada.

- Processamento de Voz: controle do transporte de voz e transporte de mídia.

Podem ser realizadas utilizando qualquer um dos protocolos da camada de transporte (UDP ou TCP). Para as aplicações de tempo real normalmente é utilizado o User Datagram Protocol (UDP), por não apresentar controle de sequência, por exemplo como uma aplicação reconstituirá a informação de voz no destino? Para este caso é necessário um protocolo auxiliar que numere os pacotes para que os mesmos possam ser reconstituídos corretamente no destino.

Diante disso, o Real-time Transport Protocol (RTP) um protocolo de transporte implementado na camada de aplicação é executado sobre o UDP e juntos são capazes de multiplexarem os diversos fluxos de comunicações multimídea, sobre um único fluxo de pacotes UDP.

Para monitorar a qualidade e a confiabilidade desse serviço o RTP também utiliza um outro protocolo o Real Time Control Protocol (RTCP) é "baseado no envio periódico de pacotes de controle a todos os participantes da conexão (chamada), usando o mesmo mecanismo de distribuição dos pacotes de mídia (Voz)" (TELECO, 2007). A principal função é fornecer feedback das informações de propriedades de redes. Portanto, o ambiente ideal: VoIP + UDP + RTP/RTCP + QoS.

\subsection{O protocolo SIP}

Para Internet ser compreendida como a "rede de redes" que conhecemos, permitindo diversos tipos de aplicações para diferentes áreas são necessários normas de regularização, são protocolos e arquiteturas de redes, e para implementação de serviços baseados em VoIP também são essenciais alguns requisitos como "normas para a inicialização, estabelecimento e finalização da comunicação. Essas normas são chamadas de protocolos de comunicação" (KELLER, 2011, p. 27).

Para que a comunicação realize a telefonia IP utiliza os protocolos TCP/UDP/IP da rede como infra-estrutura para os seus protocolos de aplicação, conforme a figura 1.

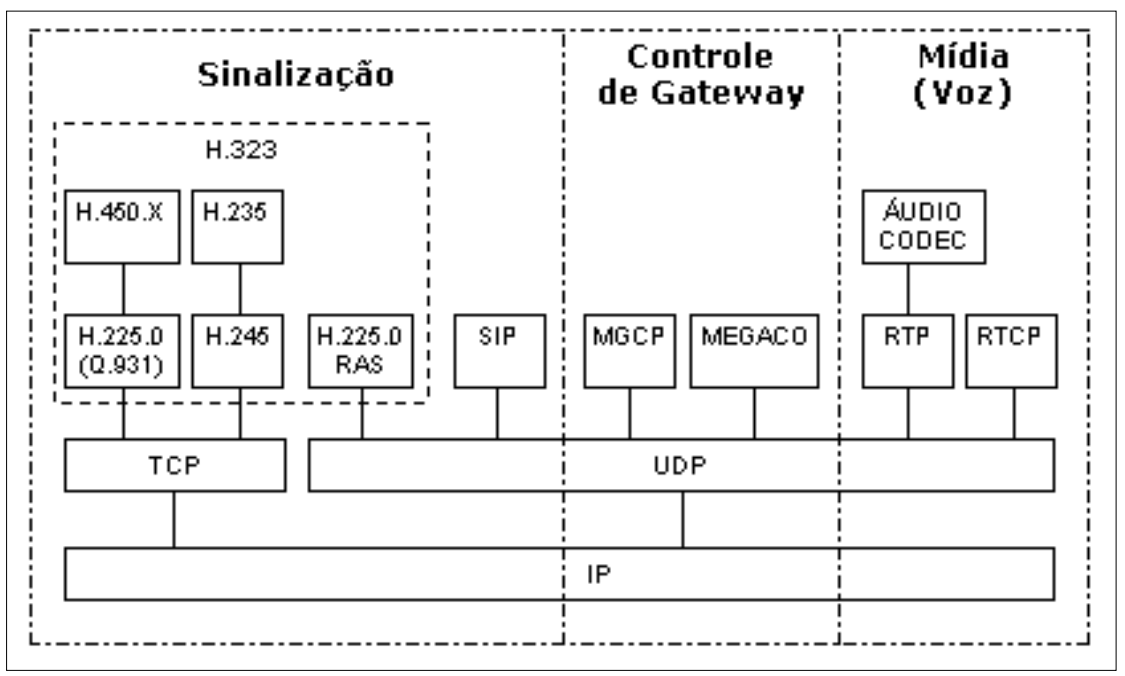

Figura 1. Estrutura em camadas dos principais protocolos. 
De acordo com Milanez (2009, p. 7), os protocolos de sinalização são "responsáveis pela criação, modificação e encerramento dos canais telefônicos virtuais existentes em chamadas VoIP. Fundamentalmente, representam o funcionamento lógico do gerenciamento dos canais físicos em ligações telefônicas que usam a comutação por pacotes". Os mais utilizados são o Inter-Asterisk eXchange (IAX) que provê sinalização e transporte de áudio, já está na segunda versão, e o Session Initiation Protocol (SIP) que permite estabelecer comunicação multimídia e proporciona facilidades de implementação e configuração, as principais características que os diferem estão na tabela 2.

Tabela 2 - As principais características dos protocolos SIP e IAX2

\begin{tabular}{|c|c|}
\hline Protocolo & Características \\
\hline \multirow{3}{*}{ SIP } & Aberto e padronizado pela IETF (rfc3261). \\
\hline & Forte adoçáo pelo mercado. \\
\hline & $\begin{array}{l}\text { Por utilizar duas portas de comunicaçáo, uma para a sinalizaçáo (UDP 5060) e outra } \\
\text { para o tráfego da mídia (RTP), o SIP apresenta algumas dificuldades e problemas na sua } \\
\text { configuraçáo quando há Network Address Translator (NAT) envolvido. }\end{array}$ \\
\hline \multirow{4}{*}{$\mathrm{IAX} 2$} & Aberto e padronizado pela IETF em fevereiro de 2010 (rfc5456). \\
\hline & Ainda tem pouca adoçáo pelo mercado. \\
\hline & $\begin{array}{l}\text { Utiliza uma porta única (UDP 4569), tanto para a sinalizaçáo quanto para o tráfego de } \\
\text { mídia, eliminando qualquer problema existente na comunicaçáo, no caso de haver NAT } \\
\text { envolvido. }\end{array}$ \\
\hline & $\begin{array}{l}\text { Em modo TRUNK, utilizado apenas para a comunicaçáo entre servidores, faz a } \\
\text { multiplexaçáo do áudio das chamadas, utilizando assim significativamente menos } \\
\text { banda para o transporte do áudio de um servidor para outro. }\end{array}$ \\
\hline
\end{tabular}

O protocolo SIP definido através da recomendação RFC 2543 do Internet Engineering Task Force (IETF), possui uma estrutura de rede dividida em clientes e servidores, realizando comunicação por troca de mensagens que podem ser requisições ou respostas, e tem seus próprios mecanismos de segurança e confiabilidade.

\section{Metodologia}

Para a realização deste minicurso são necessários os procedimentos metodológicos, primeiro é a implementação do servidor elastix, segundo é a configuração. E para finalizar, é necessário fazer testes utilizando um emulador para ligações VoIP.

\subsection{Implementando o servidor elastix}

Segundo Claudemir (2012) “o Elastix é uma distribuição baseada no CentOS (distribuição Linux muito utilizada para servidores) e integra esta opção funcional para implementar uma central telefônica digital (PBX)". E para criar este servidor o processo dar-se-á da seguinte maneira: preparar o ambiente, conforme figura 2, configurar corretamente e finalizar com um ambiente web.

Na figura 3, o servidor elastix está criado e pronto para uso. Então, utilizando o comando dhclient o servidor disponibilizará um endereço IP criado pelo Dynamic Host Configuration Protocol (DHCP), este protocolo de serviço TCP/IP oferece configuração 
dinâmica de terminais, com concessão de endereços IP. A porta padrão é a UDP 67 onde o cliente deve transmitir e receber as informações, conforme a figura 4 .

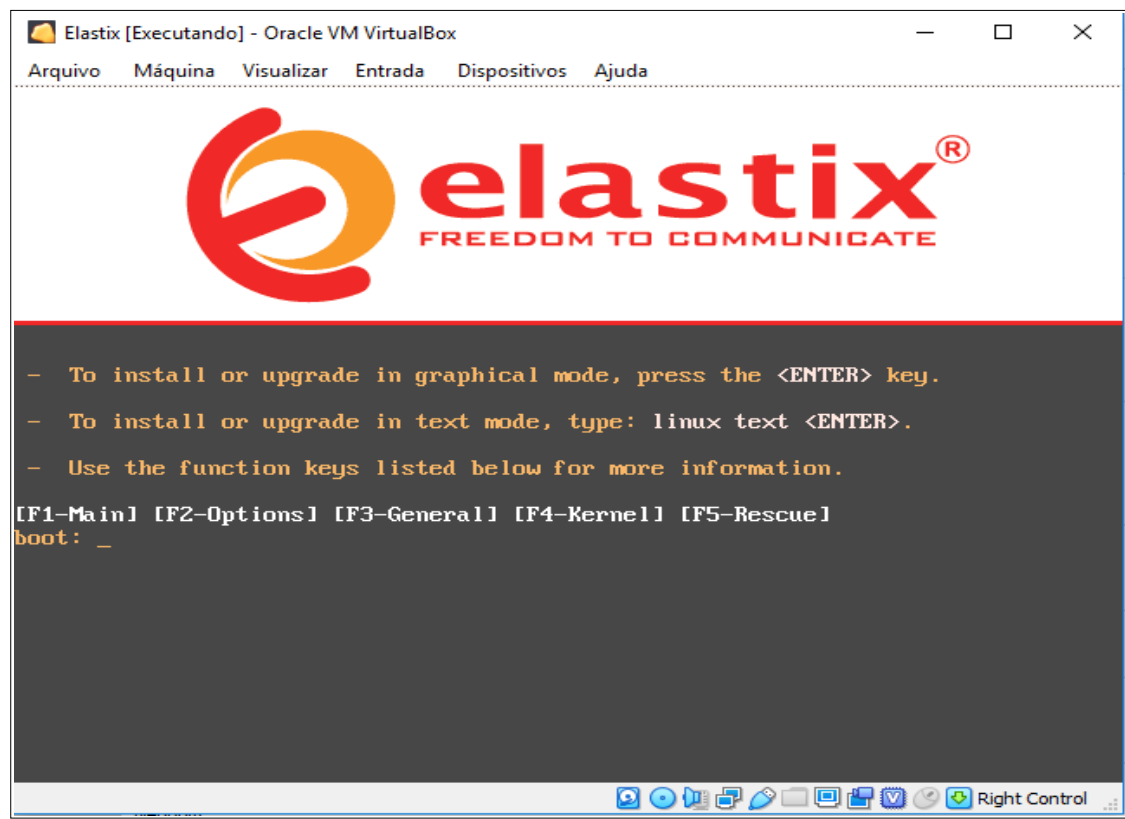

Figura 2. Preparando o ambiente para criação do servidor elastix.

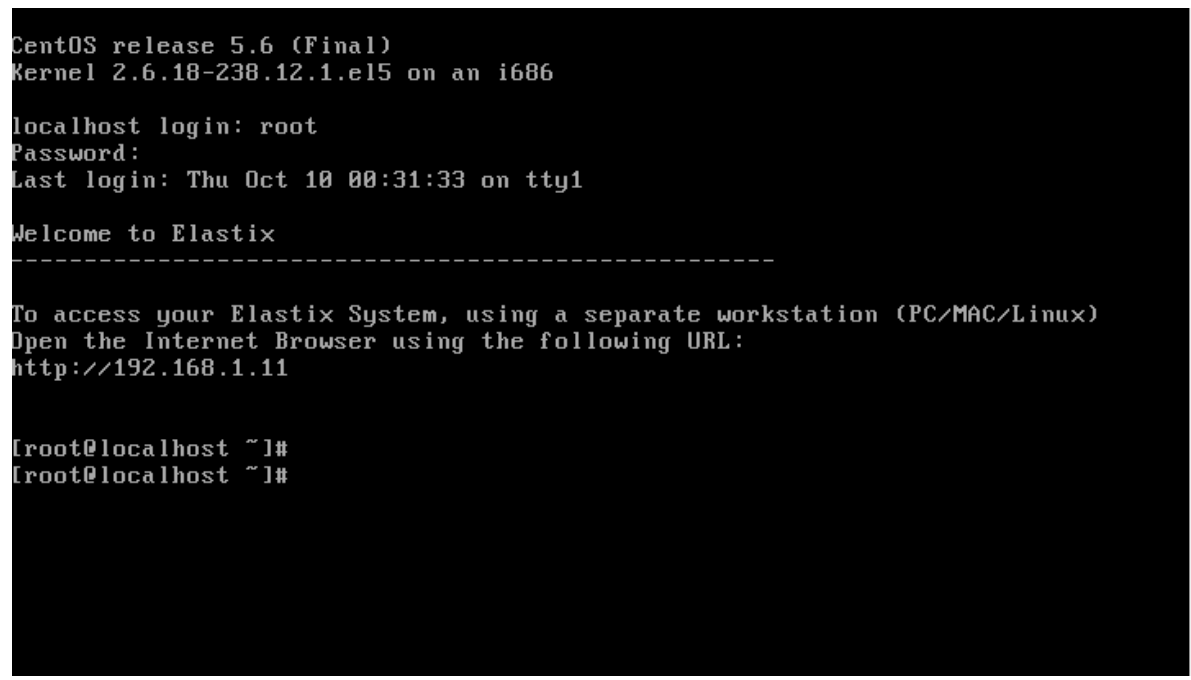

Figura 3. Servidor elastix criado. 


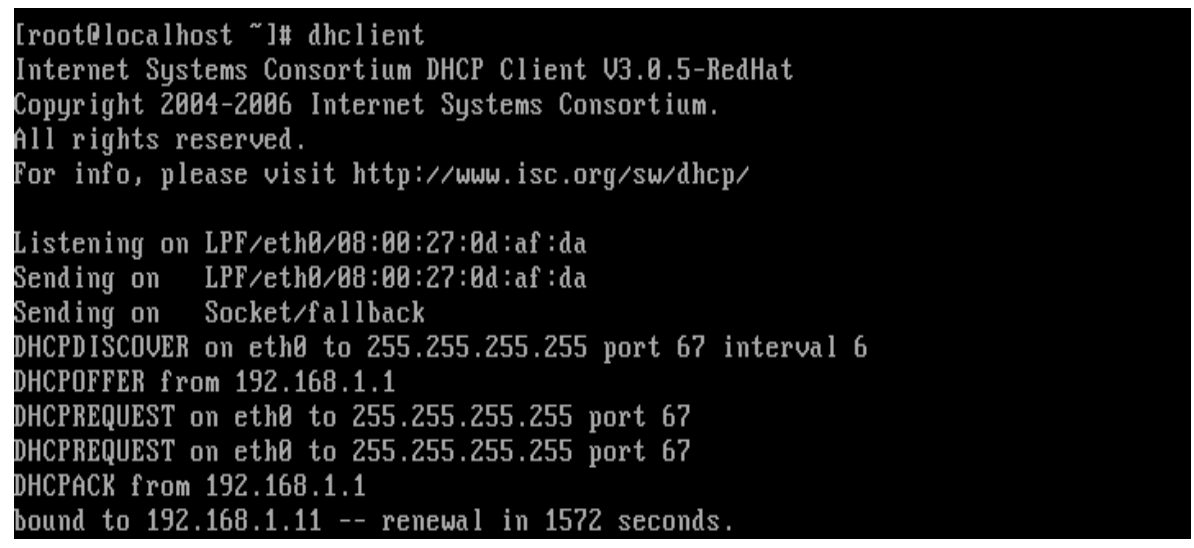

Figura 4. Utilizando o comando dhclient.

Após o procedimento de concessão de endereços IP é necessário acessá-lo para então, utilizar dos recursos disponíveis, como mostra a figura 5 é uma interface web desse servidor.

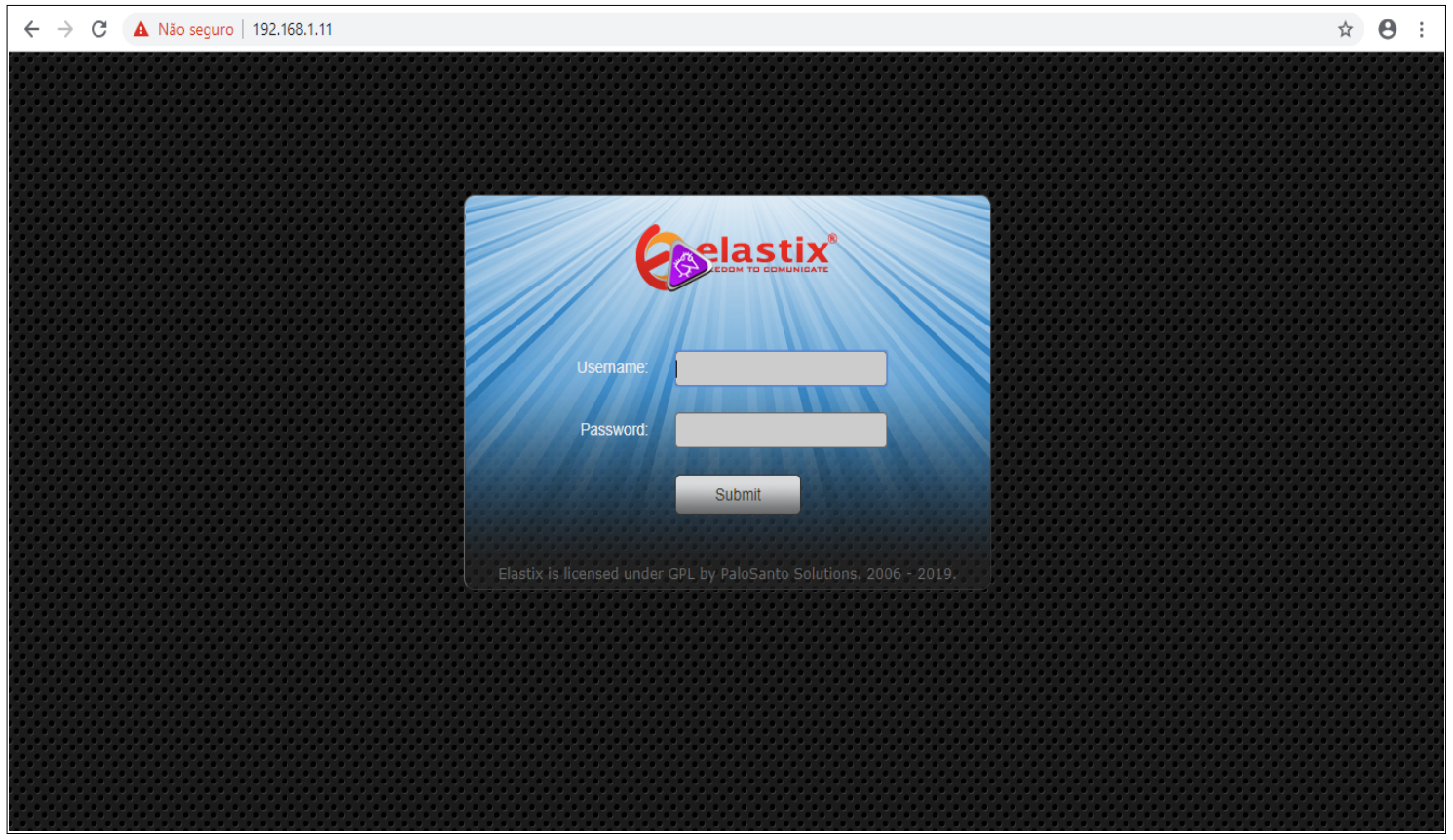

Figura 5. Servidor elastix com interface web.

\subsection{Preparando o Softphone}

Para a parte de preparar o ambiente para receber as ligações telefônicas, utilizou-se um software que emula um telefone VoIP o "Softphone é um programa ou aplicativo multimídia que via VoIP, pode ser utilizado para realizar chamadas telefônicas e vídeo chamadas através da internet, sem a necessidade de um aparelho telefônico convencional ou móvel" (FaleMaisVOIP, 2016).

Escolheu-se para este trabalho o Softphone X-lite da CounterPath por ser baseado em padrões abertos e utilizar o protocolo SIP, e também por apresentar infinidades de "opções de comunicação, segurança e criptografia superiores e codecs de áudio avançados. Os clientes de smartphones VoIP móveis do CounterPath são os 
aplicativos mais confiáveis e repletos de recursos do mercado para o Apple iOS e Android" (CounterPath, 2018).

Também possui versão para o sistema operacional windows com facilidade para personalização e variedades de provedores SIP, permiti comunicação utilizando o $3 \mathrm{G}$, Wi-fi, chamadas por vídeo, chat e compartilhamento de imagens e arquivos.

\section{Resultados Esperados}

Os resultados alcançados são divididos em duas etapas: uma para a configuração dos ramais no servidor e outra para preparação do ambiente para realização das chamadas telefônicas VoIP.

\subsection{Criando os ramais no servidor}

A figura 6 , representa a interface onde são realizados a criação e configuração dos ramais no servidor.

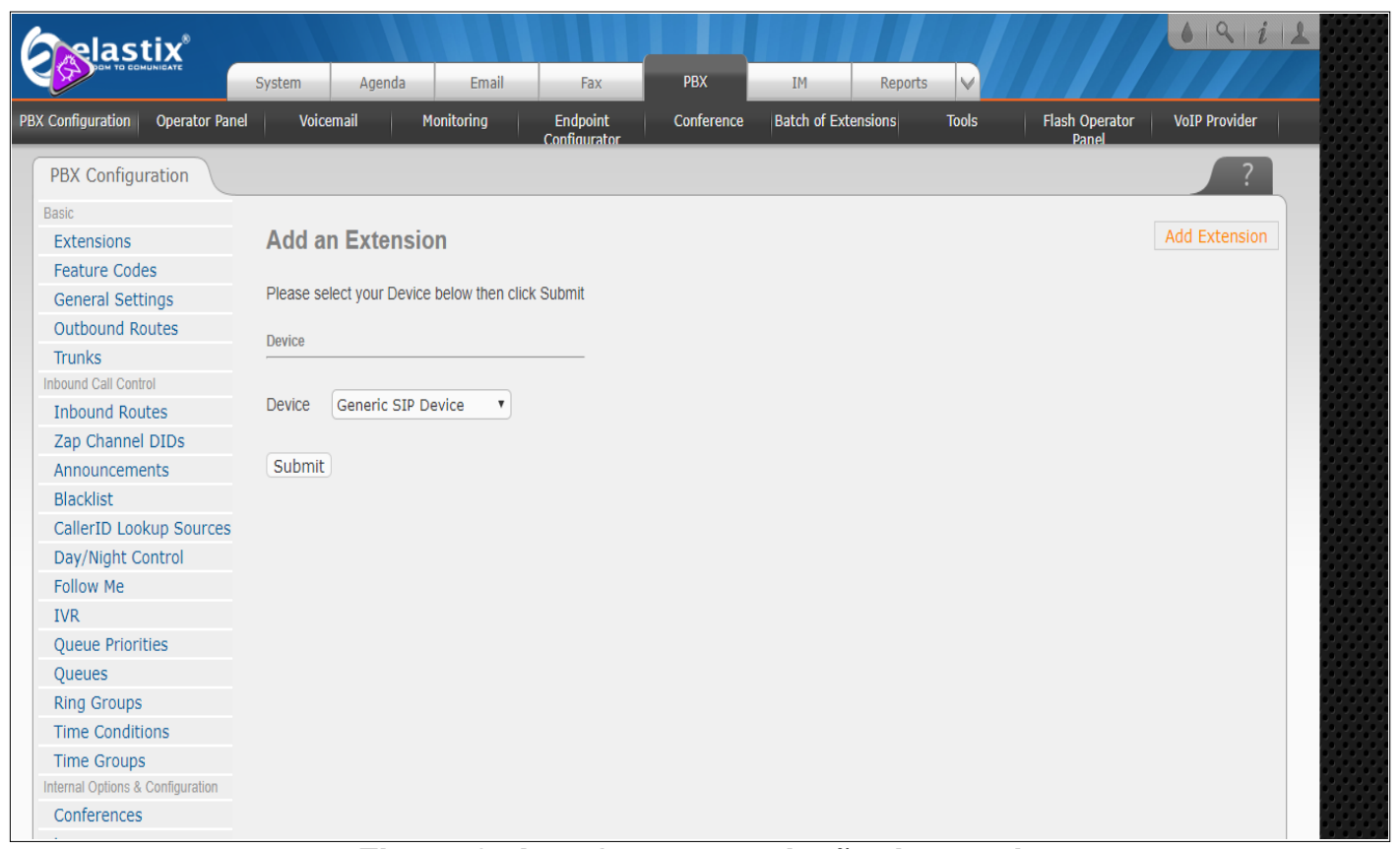

Figura 6. Interface para criação de ramal.

Foram criados dois ramais um chamado de Comercial (3000) e outro SuporteTI (3001), como mostra a figura 7. 


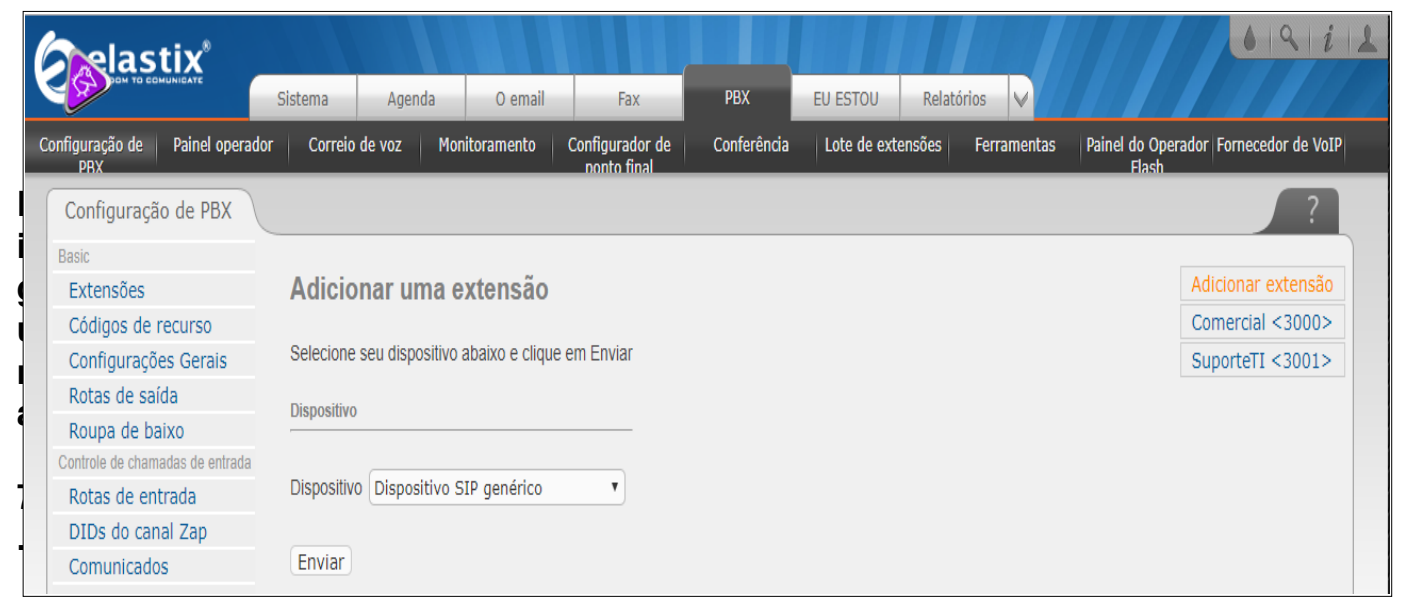

Figura 7. Ramais criados.

\subsection{Preparando o ambiente para emular as chamadas telefônicas VoIP}

O softphone utilizado para realizar as chamadas telefônicas VoIP é o X-lite e sua configuração é mostrado na figura 8 , é necessário inserir o endereço IP do servidor, seguido das configurações utilizadas para a criação do ramal como o ID de usuário e a senha escolhida. A sua instalação foi feita utilizando um computador de sistema operacional windows.

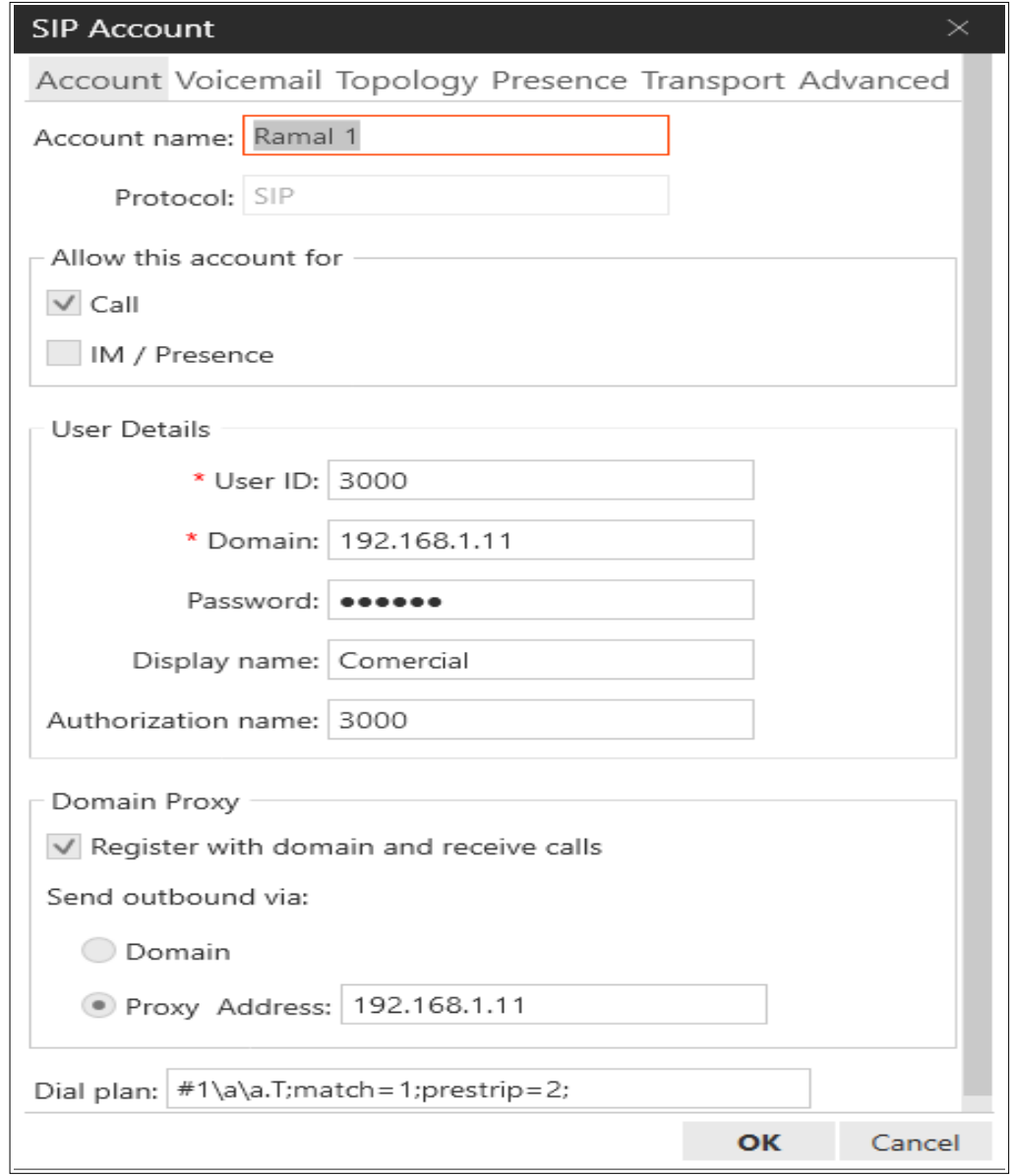

Figura 8. Preparando o ambiente de chamadas no computador. 


\section{Considerações Finais}

Neste minicurso, pudemos conhecer e explorar sobre este ambiente de telecomunicações que utilizam diversos recursos da Internet. Dessa maneira, conseguimos entender os seus principais fundamentos e recursos, também a implementação do servidor para assim, trabalhar as diversas ferramentas que ele possui.

Contemplamos a criação e configuração de ramais neste servidor, utilizamos um softphone para realizar as chamadas telefônicas e assim, verificar como todo este aparato junto podem convergir para didáticas voltado para o ensino.

\section{Agradecimentos}

Eu agradeço ao professor Dr. Ruy de Oliveira, meu professor no $9^{\circ}$ semestre na disciplina de Redes de Computadores II do curso de Engenharia da Computação e este minicurso é uma junção de um trabalho sobre o mesmo tema apresentado em sala de aula ainda durante a graduação. Foi pela didática por ele utilizada nas aulas, que aprendi a buscar sempre fazer o melhor. Hoje, tenho outra visão da academia. E posso afirmar que em grande parte eu aprendi nos últimos anos da faculdade.

\section{Referências}

BORDIM, Jacir L. (2010) "Introdução à Voz sobre IP e Asterisk", p. 11-16, 02 de abr. de 2018.

CARVALHO, Priscila F. (2018) "Modelagem de Negócios: Uma Aplicação na Implantação de um Sistema VoIP para Fornecimento de Serviços de Telefonia IP por uma Empresa da Região de Campos dos Goytacazes.” Rio de Janeiro, p. 12, 05 de nov. de 2008.

CLAUDEMIR. (2012) BLOG DOS CURSOS. "ELASTIX. Parte 2: Configurando Extensões e SoftPhone X-lite". Disponível em: http://blogdoscursos.com.br/elastixparte-2-configurando-extensoes-e-softphone-x-lite/>.

CounterPath. (2018) "Comunicações móveis em um nível totalmente novo". Disponível em: < http://www.counterpath.com/softphone-clients/>.

FaleMaisVOIP. (2016) "Softphone: o que é, como funciona e quais as opções?" Disponível em: < https://www.falemaisvoip.com.br/blog/softphone/>.

KELLER, Alexandre. (2011) "Asterisk na prática”. p. 20 - 27, 2ª edição. São Paulo: Novatec Editora, 2011.

MILANEZ, Mateus Godoi. (2009) "Avaliação dos protocolos VoIP SIP e IAX utilizando simulação e parâmetros de qualidade de voz". Dissertação (Mestrado) - Instituto de Ciências Matemáticas e de Computação. Universidade de São Paulo, USP. São Carlos/SP. 2009, p. 7.

TELECO, Inteligência em Telecomunicações. "PTT no Celular II: Protocolos SIP e RTP". Disponível em: < http://www.teleco.com.br/tutoriais/tutorialpushtotalk2/pagina_3.asp>.

TELECO, Inteligência em Telecomunicações. (2019) "VoIP/Telefonia IP”. Disponível em: $<$ http://www.teleco.com.br/voip.asp $>$. 
TELECO, Inteligência em Telecomunicações. (2019) "Telefonia IP: Protocolos". Disponível em: $<$ https://www.teleco.com.br/tutoriais/tutorialtelip/pagina_3.asp>. 